(Aus der Abteilung für Chemotherapie des Instituts für Infektionskrankhciten „Robert Koch".)

\title{
Depressionsimmunität bei intravenöser Superinfektion mit Streptokokken.
}

\author{
Von
}

\section{J. Morgenroth und I. Abraham.}

Daß die Immunität gegen Superinfektion im Falle der T u berk ulose auch bei direkter Einverleibung des zur Nachinfektion dienenden Virus in die Blutbahn unzweideutigen Ausdruck findet, ist von verschiedenen Autoren festgestellt. Die Versuche, auf die wir hier nicht näher einzugehen brauchen, sind quantitativ begründet; sie zeigen in einheitlicher Weise, daß beim Bestehen einer geringgradigen oder relativ langsam verlaufenden tuberkulösen Infektion der Versuchstiere eine bei gesunden Kontrolltieren verhältnismäßig rasch zum Tode führende intravenöse oder intrakardiale Nachinfektion die vorinfizierten Tiere anscheinend unberührt läßt. Den Versuchen von Calmette und $\mathrm{G}$ uéri $\mathbf{n}^{\mathbf{1}}$ ) an Rindern entsprechen Versuche von $\mathrm{Neu}$ feld ${ }^{2}$ ), die schon vor diesen (1904) ausgeführt, aber erst jetzt von ihm mitgeteilt wurden. Hieran schließen sich analoge Versuche von Rö mer und $\mathrm{Jose}_{\mathrm{ph}}{ }^{3}$ ) an Schafen.

Neuerdings wurden diese Versuche durch Debré und Paraf ${ }^{4}$ ) auch an Meerschweinchen bestätigt. In einer großen Versuchsreihe waren $78 \%$ der Versuche erfolgreich. Wir bezweifeln nicht, daß auch bei den größeren Versuchstieren ähnliche Ausfälle des Erfolges zu beobachten wären, wenn man an großen Versuchsreihen arbeiten könnte. Die prinzipielle Bedeutung des Phänomens würde dadurch nicht beeinträchtigt.

Nachdem wir unsere Anschauungen über Depressionsimmunität ${ }^{5}$ ) auf die Erscheinungen bei der Su perinfektion mit Streptokokken stützen und vorläufig in ihr das hauptsächliche Feld für experimentelle Betätigung in dieser Richtung sehen müssen, erschienen Untersuchungen

1) Calmette und Guérin, Ann. Inst. Pasteur 1908; 22, S. 689.

2) Ne ufeld, Zeitschr. f. Tuberkul. 34, 312. 1921.

3) Römer und Jose ph, Beitr. z. Klin. d. Tuberk. 17, 287. 1910.

4) Debré und Paraf, C. R. Soc. Biol. 83, 1920, S. 1598, ref. Bull. Inst. Pasteur 1921, S. 103.

5) Morgenroth, Biberstein u. Schnitzer, Dtsch. med. Wochenschr1920, Nr. 13; Schnitzer und v. Kühlewein, Zeitschr. f. Hyg. u. Infektionskrankh. 92, 492. 1921 . 
über intravenöse Nachinfektion mit Streptokokken geboten. Wir hatten von Anfang an angenommen, daß hier grundsätzlich dieselben Gesetzmäßigkeiten statthaben wie in dem klassischen Versuch Robert Kochs am tuberkulösen Meerschweinchen. Entsprechende Analogien waren auch bei intravenöser Nachinfektion mit Streptokokken zu erwarten.

Nach dem von uns vorausgesetzten kinetischen Charakter der Depressionsimmunität durfte man von vornherein nicht mit sehr großen Ausschlägen rechnen. Dazu kam noch, daß wir, wie aus den folgenden Protokollen zu ersehen ist, zur Erzielung einer gleichmäßigen intravenösen Nachinfektion vielfach hohe, zum Teil sehr hohe Multipla der tödlichen Dosis angewendet haben. Es ist auch durchaus nicht sicher, ob die von uns benutzten Intervalle gerade die günstigsten waren. Wie dem aber auch - sei, ist es nicht zu bezweifeln, daß auch hier eine prinzipielle Übereinstimmung mit dem Verhalten der Tuberkuloseinfektion besteht.

Es dürfte angezeigt sein, über sämtliche von uns ausgeführten Versuche im folgenden zu berichten, da auch die minder gelungenen für die Beurteilung der gesamten Sachlage nicht ohne Bedeutung sind.

Wir haben im ganzen 15 Versuchsreihen ausgeführt; eine derselben kommt in Wegfall, weil durch einen plötzlichen Virulenzanstieg des. Vorinfektionsstammes sämtliche Tiere vor erfolgter Nachinfektion starben. Eine zweite Reihe, bei der nach unserem Ermessen eine erhebliche Immunität zum Ausdruck kam, haben wir nicht in diese Darstellung aufgenommen, da der verzögerte Tod einer Kontrolle nicht abgewartet, sondern das Tier irrtümlicherweise getötet wurde.

Hieran schließen sich fünf weitere Versuche, die wir deshalb nicht ausführlich schildern, weil keines der vorinfizierten Tiere die zahlreichen und pünktlich gestorbenen Kontrollen um mehr als einen Tag überlebte. Das Überleben um einen Tag traf in den verschiedenen Versuchen für 10\% (Intervall 2 Tage), 20\% (Intervall $1 \mathrm{Tag}$ ), 25\% (Intervall 3 Tage), 25\% (Intervall 3 Tage) und 57\% (Intervall 1 Tag) der Versuchstiere zu. Auch diese Ergebnisse sind unseres Erachtens als positive anzusehen, immerhin nähern sie sich zu stark den Fehlergrenzen, als daß sie eine besondere Betonung beanspruchen dürften.

Wir glauben uns zu der Behauptung berechtigt, daß in 14 Versuchsreihen niemals ein völlig negatives Resultat erzielt wurde. Dabei ist immer noch zu erwägen, daß stets eine scheinbare Verschlechterung der Versuchsergebnisse dadurch zum Ausdruck kommen kann, daß einzelne Tiere, deren Tod der Nachinfektion zur Last gelegt wird, tatsächlich der Vorinfektion erlegen sind, was im Einzelfall weder auszuschließen, noch, wenn nicht etwa die Vorinfektion mit grünen. Streptokokken stattfand, überhaupt zu beurteilen ist. 
Die acht Versuche, die nun noch übrigbleiben, seien im folgenden durch Protokolle und Tabellen zur Anschauung gebracht.

Über die Versuchstechnik ist wenig zu sagen. Es wurden stets 18-24stündige Kulturen in 10 proz. Pferdeserumbouillon verwendet, die mit dem gleichen Medium entsprechend verdünnt wurden. Die Abimpfung der Tiere erfolgte in der Weise, daß wir zunächst den Thorax steril öffneten und aus der Pleurahöhle mit dem Glasspatel abimpften. Dann entnahmen wir Herz und Lunge und führten die frisch angelegten Schnittflächen über die Blutagarplatte. Ebenso verfuhren wir mit den Bauchorganen (Milz, Leber, Niere), nachdem wir aus dem Peritoneum mit dem Glasspatel abgeimpft hatten. In einem Versuch wurde auch aus der Harnblase abgeimpft.

\section{Versuch 1.}

Vorinfektion intraperitoneal mit dem grünen Str. 890 0,3 Vollkultur.

Nachinfektion intravenös am folgenden Tag mit Str. H. II 0,3 1/1090.

Der von Anfang an grüne Stamm 890 war von einer Diphtherieplatte gezüchtet. 0,3 Vollkultur intraperitoneal tötete nicht akut.

Der Stamm H II ist ein hochvirulenter, vom Menschen stammender Streptokokkus, ohne Tierpassage, der schon von Schnitzer und Munter*) beschrieben wurde. Er wird seit dem 16. VI. 1920 in unserem Laboratorium geführt und hatte seitdem eine konstante Virulenz bei peritonealer und subcutaner Infektion.

0,3 $1: 10$ Millionen intraperitoneal und subcutan töteten in 48 Stunden.

Zahlreiche Vorversuche mit in tra. venöser Infektion des Stammes ergaben eher geringere Virulenz. 0,3 1: 10 Millionen und 1:1 Million tötete fast regelmäßig, aber erst nach 3 Tagen, mit $0,31: 10000$ and 1:5000 blieben einmal 2 Tiere 6 Tage am Leben. 0,3 1:3000 und 1: 2500 tötete gelegentlich erst nach 4-5 Tagen. Wir mußten also der Sicherheit halber zu der ungemein hohen Dosis von 0,3 $1: 1000$ übergehen, die fast regelmäßig in $1-2$ Tagen, sehr selten in 3 Tagen tötete. $\mathrm{V}=$ Vorinfektion, $\mathrm{S}=$ intravenöse Superinfektion, $\mathrm{D}=$ diagnostisch getötet.

Zu dem Versuch ist zu bemerken, daß hier ein äußerst wenig virulenter, nicht selten versagender, grüner Stamm zur Vorinfektion diente, während die Anforderungen bei der Nachinfektion durch die sehr große Infektionsdosis des hochvirulenten Stammes H II außerordentlich groß waren.

\begin{tabular}{|c|c|c|c|c|c|}
\hline \multicolumn{6}{|c|}{ Tag } \\
\hline 0 & 1 & 2 & 3 & 4 & 5 \\
\hline$V$ & $\mathrm{~S}$ & \multirow{14}{*}{$\dagger$} & $\left.\dagger^{1}\right)$ & . & \multirow{14}{*}{$\begin{array}{l}\left.f^{-4}\right) \\
\left.f^{1}\right)\end{array}$} \\
\hline $\mathrm{V}$ & $S$ & & $\left.\dot{r}^{-1}\right)$ & & \\
\hline $\mathrm{V}$ & $S$ & & $\left.r^{2}\right)$ & & \\
\hline$V$ & $\mathbf{S}$ & & $\left.f^{1}\right)$ & & \\
\hline $\mathrm{V}$ & $S$ & & $t$ & & \\
\hline V & $S$ & & +3 & & \\
\hline $\begin{array}{l}\mathrm{V} \\
\mathrm{V}\end{array}$ & $\begin{array}{l}S \\
S\end{array}$ & & 11) & & \\
\hline$V$ & $\mathrm{~S}$ & & & & \\
\hline- & $\mathbf{S}$ & & & & \\
\hline 一. & $\mathrm{S}$ & & & & \\
\hline- & $S$ & & $t$ & & \\
\hline$\dot{I}$ & $\begin{array}{l}S \\
S\end{array}$ & & 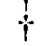 & & \\
\hline$V$ & $\left.D^{5}\right)$ & & & & \\
\hline $\mathbf{V}$ & $\left.D^{5}\right)$ & & & & \\
\hline
\end{tabular}

1) Peritoneum, Organe, Harnblase +++ hämolytische Str.

2) Harnblase ++ hämolytische + grüne Str.

3) Peritoneum, Organe +++ hämolytische Str. Harnblase ++ hämolytische Str. (+) grüne Str.

4) Peritoneum, Pleura, Organe +++ hämolytische $(+)$ grüne Str.

5) Peritoneum, Organe +++ grüne Str.

*) Schnitzer und Munter, Zeitschr. f. Hyg. u. Infektionskrankh. 94, 107: 1921. 
Versuch 2 und 3.

Versuch 2. Vorinfektion intravenös mit dem hämolytischen Str. E 0,4 1/100. Nachinfektion intravenös nach 2 Tagen mit Str. E ,Passage " 0,3 Vollkultur.

Versuch 3. Vorinfektion intravenös mit Str. E 0,4 1/100.

Nachinfektion intravenös nach 2 Tagen mit Str. E ,Passage " 0,3 1/10.

Stamm E war wenig virulent; 0,3 1/10 tötete bei intraperitonealer Infektion innerhalb 24 Stunden, bei intravenöser Infektion töteten 0,3 Vollkultur nur einen Teil der Tiere in 24 Stunden, die übrigen in 2-3 Tagen.

Der zur Nachinfektion dienende Stamm E "Passage" war nach zahlreichen Mäusepassagen virulenter als Stamm E. 0,3 Vollkultur tötete bei intravenöser Infektion mit Sicherheit in 24 Stunden, geringere Dosen wurden früher nicht ausprobiert.

Tabelle II.

\begin{tabular}{|c|c|c|c|c|c|c|c|c|c|c|}
\hline \multicolumn{11}{|c|}{ Tag } \\
\hline 0 & 1 & 2 & 3 & 4 & 5 & 6. & 7 & 8 & 9 & 10 \\
\hline V & & $\mathbf{S}$ & $\dagger$ & & & & & & & \\
\hline V & & $S$ & & $\dot{\dagger}$ & & & & & & \\
\hline V & & $\mathrm{S}$ & & $\dagger$ & & & & & & \\
\hline $\mathrm{V}$ & & $\mathrm{S}$ & & $\dagger$ & & & & & & \\
\hline$V$ & & $\mathrm{~S}$ & & & $i$ & & & & & \\
\hline- & & $S$ & $\doteqdot$ & & & & & & & \\
\hline- & & $\mathrm{S}$ & $\dagger$ & & & & & & & \\
\hline 一 & & $\mathbf{S}$ & $\dagger$ & & & & & & & \\
\hline - & & S & $\dagger$ & & & & & & & \\
\hline- & & $\mathbf{S}$ & $\dagger$ & & & & & & & \\
\hline V & & $\left.D^{1}\right)$ & & & & & & & & \\
\hline V & & $\left.\mathrm{D}^{2}\right)$ & & & & & & & & \\
\hline V & & & & & & & & & $\left.D^{3}\right)$ & \\
\hline $\begin{array}{l}V \\
V\end{array}$ & & & & & & & & & & $D^{5}$ \\
\hline & & & & & & & & & & \\
\hline
\end{tabular}

1) Niere ++ , Peritoneum, Pleura, Organe 0 .

2) Peritoneum, Pleura, Organe $(+-)$.

3) Herzblut, Pleura, Niere +++ , Lunge ++ , Milz 0 .

4) Peritoneum, Pleura und Organe 0.

5) Niere +++ , Pleura, Lunge, Herzblut $(+)$, Peritoneum, Milz 0.

Tabelle III.

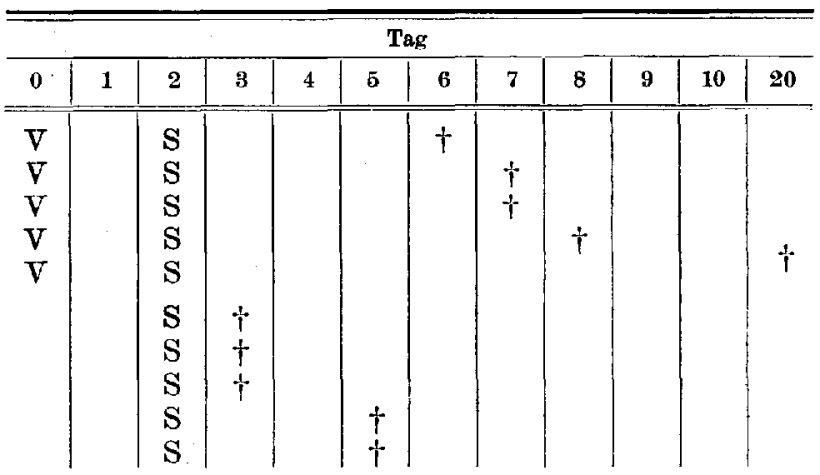



such 2 .

Die Vorinfektionskontrollen zu Versuch 3 sind die gleichen wie bei Ver-

Die Versuche unterscheiden sich bei gleicher Vorinfektion durch die verschieden starke Nachinfektion. Der schwächeren, nach den Kontrollen durchaus ausreichenden Nachinfektion entspricht längeres Überleben der Tiere. Eine der Vorinfektionskontrollen ergab am 9. Tage kein Wachstum melr.

\section{Versuch 4 und 5.}

Versuch 4. Vorinfektion subcutan mit Str. $150,21 / 10$.

Nachinfektion intravenös nach 3 Tagen mit Str. H. II 0,3 1/250.

Versuch 5. Vorinfektion subcutan mit Str. 15 0,2 1/10.

Nachinfektion intravenös nach 3 Tagen mit Str. H. II $0,31: 2000$,

Von Str. 15 tötete $0,31 / 10$ und 0,3 1/100 intraperitoneal in 24 Stunden, $0,31 / 1000$ in 48 Stunden. $0,21 / 10$ und $0,21 / 100$ machte subcutan typische Phlegmone.

Bezüglich Str. H II s. Versuch 1.

Tabelle IV.

\begin{tabular}{|c|c|c|c|c|c|c|c|c|c|}
\hline \multicolumn{10}{|c|}{ Tag } \\
\hline 0 & 1 & 2 & 3 & 4 & 5 & 6 & 7 & 8 & 9 \\
\hline V & & & $\mathbf{S}$ & & + & & & & \\
\hline $\mathrm{V}$ & & & $S$ & & 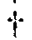 & & & & \\
\hline$V$ & & & $\mathbf{S}$ & & $\uparrow$ & & & & \\
\hline$V$ & & & $\mathrm{~S}$ & & & $\dagger$ & & & \\
\hline$V$ & & & $S$ & & & $\dot{\gamma}$ & & & \\
\hline $\mathrm{V}$ & & & $\mathbf{S}$ & & & $\dot{\varphi}$ & & & \\
\hline V & & & $\mathrm{S}$ & & & 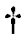 & & & \\
\hline V & & & $\mathbf{S}$ & & & + & & & \\
\hline $\mathbf{V}$ & & & $\mathbf{S}$ & & & & $\dagger$ & & \\
\hline V & & & $\mathbf{S}$ & & & & & & $\dagger$ \\
\hline 一 & & & $\mathrm{S}$ & + & & & & & \\
\hline- & & & $\mathrm{S}$ & $\dagger$ & & & & & \\
\hline- & & & $\mathrm{S}$ & $\forall$ & & & & & \\
\hline- & & & $\mathbf{S}$ & $\uparrow$ & & & & & \\
\hline- & & & $\mathbf{S}$ & $\dagger$ & & & & & \\
\hline- & & & $\mathrm{S}$ & & $\dagger$ & & & & \\
\hline- & & & $\mathbf{S}$ & & $\nrightarrow$ & & & & \\
\hline- & & & $\mathbf{S}$ & & $\uparrow$ & & & & \\
\hline- & & & $\mathrm{S}$ & & + & & & & \\
\hline- & & & $\mathbf{S}$ & & $\dagger$ & & & & \\
\hline 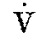 & & & $\left.D^{1}\right)$ & & & & & & \\
\hline$\nabla$ & & & $\left.D^{1}\right)$ & & & & & & \\
\hline$\forall$ & & & & & & & $\left.D^{1}\right)$ & & \\
\hline$\nabla$ & & & & & & & $\left.D^{1}\right)$ & & \\
\hline V & & & & & & & $\left.D^{2}\right)$ & & \\
\hline
\end{tabular}

1) Subcutangewebe, Peritoneum, Pleura, Organe +++ . Niere 0.

2) Subcutangewebe, Lunge +++ , Peritoneum + , Pleura, Herzblut, Milz, 
Tabelle V.

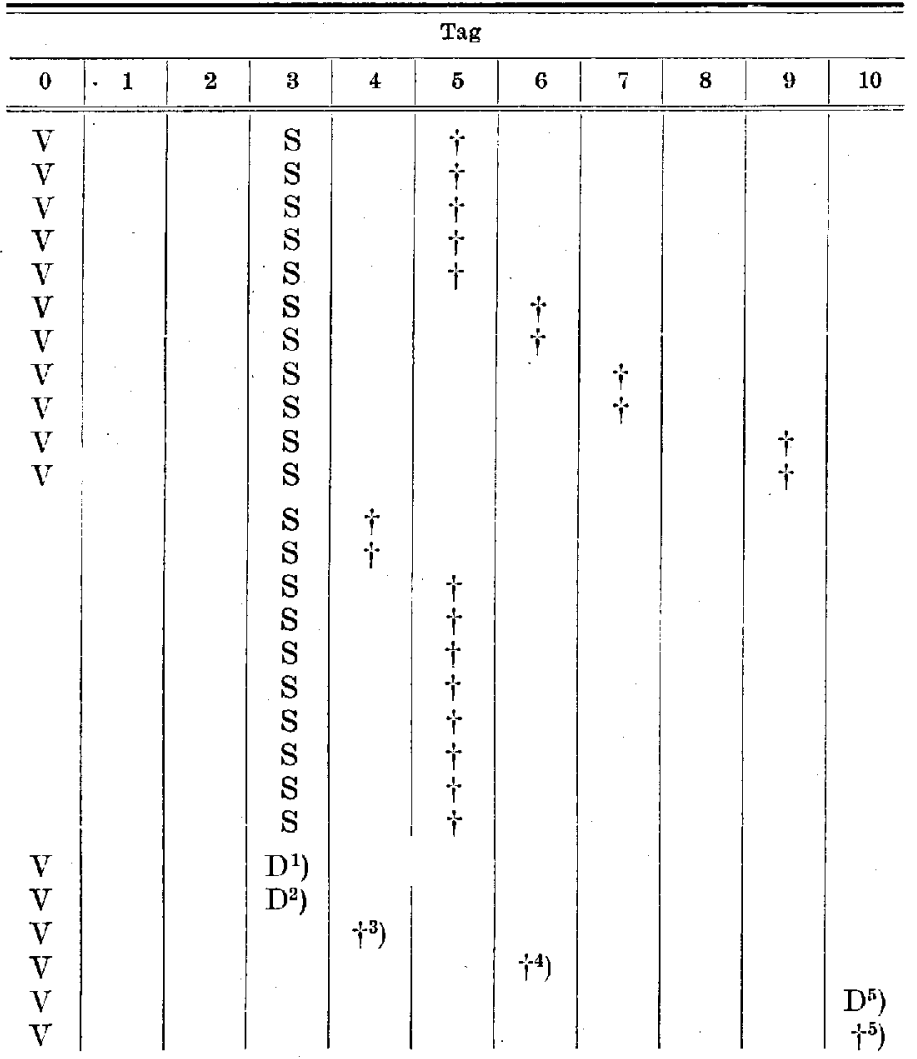

1) Subcutangewebe, Peritoneum, Pleura, Organe +++ .

2) Subcutangewebe, Peritoneum, Pleura, Herzblut, Lunge,+++ Milz, Niere + .

3) Subcutangewebe, Pleura, Organe +++ , Peritoneum + .

4) Nicht seziert.

5) Subcutangewebe, Peritoneum, Pleura, Organe +++ .

Die Vorinfektionen mit dem Str. 15 verlaufen gleichmäßig. Bei Versuch 4 ist die zur Nachinfektion verwendete Dosis des Str. H II eine exorbitant hohe.

Versuch 6 und 7.

Versuch 6. Vorinfektion subcutan mit Str. $8760,31 / 500$.

Nachinfektion intravenös nach 3 Tagen mit Str. 876 0,3 1/10.

Versuch 7. Vorinfektion subcutan mit Str. $8760,21 / 500$.

Nachinfektion intravenös nach 3 Tagen mit Str. $8760,31 / 2$.

Der Stamm 876 wurde bereits von Schnitzer und Munter*) verwendet. Intraperitoneal tötete 0,3 1/250 und 0,3 1/500 zwei Drittel der Versuchstiere in 24 Stunden. Subcutan wurde mit 0,2 1:800000! noch Phlegmone erzeugt. Intravenös tötete 0,3 Vollkultur, $0,3^{1} / 2,0,3 \frac{1}{5}$ und $0,3^{1 / 10}$ in 24 Stunden, $0,3^{1 / 100}$ und $0,31 / 1000$ in 48 Stunden. Weitere Verdünnungen wurden nicht eingestellt.

*) Schnitzer und Mu ter, Zeitschr. f. Hyg. u. Infektionskrankh. 93, 96. 1921. 
Depressionsimmunität bei intravenöser Superinfektion mit Streptokokken. 169

Tabelle VI.

\begin{tabular}{|c|c|c|c|c|c|c|c|c|c|}
\hline \multicolumn{10}{|c|}{ Tag } \\
\hline 0 & 1 & 2 & 8 & 4 & 5 & b & 7 & 8 & 9 \\
\hline $\begin{array}{l}V \\
V \\
V \\
V \\
V \\
V \\
V \\
V \\
V \\
V \\
V \\
V \\
V \\
V \\
V \\
V \\
V \\
V \\
- \\
- \\
- \\
- \\
- \\
- \\
V \\
V\end{array}$ & & & $\begin{array}{c}S \\
S \\
S \\
S \\
S \\
S \\
S \\
S \\
S \\
S \\
S \\
S \\
S \\
S \\
S \\
S \\
S \\
S \\
S \\
S \\
S \\
S \\
S \\
S \\
S \\
S \\
S \\
S^{\prime} \\
\left.D^{1}\right) \\
D^{1}\end{array}$ & $\begin{array}{l}t \\
t \\
t\end{array}$ & $\begin{array}{l}+ \\
\vdots \\
+\end{array}$ & $\begin{array}{l}+ \\
\dagger \\
t \\
+\end{array}$ & $\dot{\dagger}$ & + & 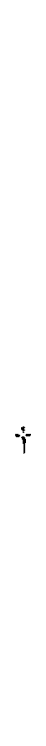 \\
\hline
\end{tabular}

Tabelle VII.

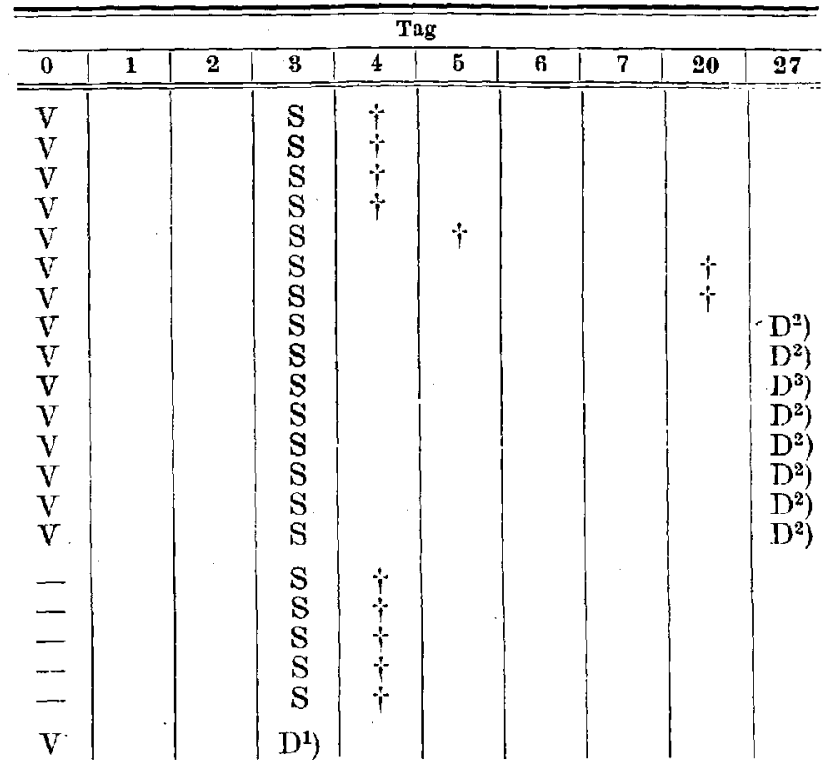

1) Subcutangewebe, Peritoneum, Pleura, Organe +++ .

2) Subcutangewebe, Peritoneum, Pleura, Organe 0.

3) Subcutangewebe, Niere, Milz, Herzblut, +++ Lunge, Leber, Pleura ++ . 
Der zur Vorinfektion verwendete Str. 876 war besonders gut für diesen Zweck geeignet. Die zur Nachinfektion verwendete Menge war die 100 resp. 500 fache der in 48. Stunden tödlichen Dosis.

Die Sektion dor dauernd überlebenden Tiere 27 Tage nach der Vorinfektion ergibt nur bei einer Maus Fortbestehen der chronischen Infektion, während dieselbe bei den übrigen 7 Tieren mit der angewandten Methode nicht mehr nachgewiesen wurdc.

\section{Versuch 8.}

Vorinfektion intravenös mit Str. E. $0,4^{1 / 100}$.

I. Nachinfektion intravenös nach 3 Tagen mit Str. E 0,3 Vollkultur.

II. Nachinfektion intravenös nach 14 Tagen mit Str. E ,Passage " 0,3 Vollkultur.

Str. E und Str. E „Passage“ s. Vorbemerkung zu Versuch 2 und 3.

Tabelle VIIL.

\begin{tabular}{|c|c|c|c|c|c|c|c|c|c|c|c|c|c|c|c|c|c|c|c|c|}
\hline \multicolumn{21}{|c|}{ Tag } \\
\hline 0 & 1 & 2 & 3 & 4 & 5 & 6 & 7 & 8 & 9 & 10 & 11 & 12 & 13 & 14 & 15 & 16 & 17 & 18 & 19 & 20 \\
\hline$Y$ & & & $S$ & & & $\dagger$ & & & & & & & & & & & & & & \\
\hline$V$ & & & $\mathbf{S}$ & & & & $i$ & & & & & & & & & & & & & \\
\hline$V$ & & & $\mathbf{S}$ & & & & & & & & & & & & & & & & & \\
\hline$V$ & & & S & & & & & & & & & & & $\mathrm{S}_{2}$ & & 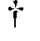 & & & & \\
\hline$V$ & & & S & & & & & & & & & & & $\mathrm{S}_{2}$ & & & $\dagger$ & & & \\
\hline $\begin{array}{l}\text { V } \\
\text { V }\end{array}$ & & & $\begin{array}{l}S \\
S\end{array}$ & & & & & & & & & & & $\mathrm{~S}_{2}$ & & & & & + & \\
\hline$\nabla$ & & & S & & & & & & & & & & & $\mathrm{S}_{2}$ & & & & & & \\
\hline- & & & $\mathbf{S}$ & & $\dagger$ & & & & & & & & & 2 & & & & & & $T$ \\
\hline- & & & S & & $\dagger$ & & & & & & & & & & & & & & & \\
\hline- & & & $\mathbf{S}$ & & $\dagger$ & & & & & & & & & & & & & & & \\
\hline- & & & S & & & $T$ & & & & & & & & & & & & & & \\
\hline- & & & - & & & & & & & & & & & $\mathrm{S}_{2}$ & $\dot{t}$ & & & & & \\
\hline- & & & - & & & & & & & & & & & $\mathrm{S}_{2}$ & $\dagger$ & & & & & \\
\hline- & & & - & & & & & & & & & & & $\begin{array}{l}\mathrm{S}_{2} \\
\mathrm{~S}_{2}\end{array}$ & $T$ & & & & & \\
\hline$V$ & & $\left.D^{1}\right)$ & - & & & & & & & & & & & & & & & & & \\
\hline$V$ & & $\left.D^{1}\right)$ & & & & & & & & & & & & & & & & & & \\
\hline $\mathrm{V}$ & & & & & & & & & & & & $\left.\dagger^{2}\right)$ & & & & & & & & \\
\hline V & & & & & & & & & & & & & & $\left.\dagger^{3}\right)$ & & & & & & \\
\hline
\end{tabular}

1) Peritoneum, Pleura, Organe 0.

2) Peritoneum, Pleura ++ , Organe + .

3) Peritoneum, Pleura, Organe +++ .

Zu diesem Versuch dienten dieselben Kulturen wie zu Versuch 2 und 3.

Auf die zweite Nachinfektion, die erhebliche Verzögerungen ergibt, ist weniger Gewicht zu legen, da die Fortdauer der Vorinfektion nach so langer Zeit nicht genügend gesichert ist.

\section{Zusammenfassung:}

Die folgende Übersichtstabelle veranschaulicht nochmals die hier geschilderten Versuche. Die Versuche 7 und 8 zeigen ein besonders gutes Ergebnis. 
Depressionsimmunitiit beì intravenöser Superinfektion mit Streptokokken. 171

\begin{tabular}{c|c|c|c|c}
\hline \hline Nr. des Versuches & Art der Vorinfektion & Intervall & Verzögert & Verzögert mehr als I Tag \\
\hline I. & Intraperitoneal & 1 Tag & $22 \%$ & $22 \%$ \\
III. & intravenös & 2 Tage & $80 \%$ & $20 \%$ \\
III. & intravenös & 2 Tage & $100 \%$ & $100 \%$ \\
IV. & subcutan & 3 Tage & $70 \%$ & $20 \%$ \\
V. & subcutan & 3 Tage & $55 \%$ & $37 \%$ \\
VI. & subcutan & 3 Tage & $85 \%$ & $60 \%$ \\
VII. & subcutan & 3 Tage & $70 \%$ & $66 \%$ \\
VIII. & intravenös & 3 Tage & $88 \%$ & $62 \%$
\end{tabular}

Die subcutane und intravenöse Vorinfektion erscheint prinzipiell in gleicher Weise brauchbar.

Was die Intervalle zwischen Vor- und Nachinfektion betrifft, so sind die Versuche nicht zahlreich genug, um hier endgültige Urteile zuzulassen.

Versuch 1 zeigt, daß die Immunität schon nach 24 Stunden vorhanden ist. Versuch 2 und 3 sind mit $z$ weitägige $m$ Intervall angestellt, Versuch 4-8 mit dreitägigem Intervall.

Schon Römer ${ }^{1}$ ) hat bezüglich der Tuberkulose beim Menschen darauf hingewiesen, daß der Erfolg eines Einbruchs von Tuberkelbacillen in die Blutbahn, analog dem Verhalten im Tierexperiment bei intravenöser Nachinfektion, unter den Gesetzen der Superinfektion steht. Das gleiche läßt sich aus unseren Versuchen für die Streptokokkeninfektion des Menschen, besonders für pyämische Erkrankungen folgern. Entsprechend unseren allgemeinen Anschauungen über Depressionsimmunität wird von Anbeginn der Infektion der Erfolg einer Blutinvasion von dem augenblicklichen Stand der Depressionsimmunität abhängen.

1. Nach subcutaner, intraperitonealer und intravenöser, chronisch verlaufender Vorinfektion mit Strep. tokokken tritt bei Mäusen innerhalb 1-3 Tagen eine Immunität gegendieakutverlaufendeintravenöse Nachinfektion mit Streptokokken ein.

2. Diese Immunität (,Depressionsimmunität“) führt zu einer mehroder weniger ausgeprägten Verzögerung oderauch zum Ausbleibendes Todes.

1) Römer, Beitr, z. Klin. d. Tuberkul. 17, 383. 1910. 